\title{
Is it possible to predict the need of inguinal lymphadenectomy in patients with squamous cell carcinoma of the penis? A clinical and a pathological study
}

\author{
Daniele Minardi ${ }^{1}$, Guendalina Lucarini ${ }^{2}$, Oriana Simonetti ${ }^{4}$, Roberto Di Primio ${ }^{2}$, \\ Rodolfo Montironi ${ }^{3}$, Giovanni Muzzonigro ${ }^{1}$ \\ ${ }^{1}$ Institute of Maternal and Children's Sciences-Urology, Polytechnic University of the Marche Region, Azienda Ospedaliero- \\ Universitaria Ospedali Riuniti, Ancona, Italy; \\ ${ }^{2}$ Department of Molecular Pathology and Innovative Therapies-Histology, Polytechnic University of the Marche Region, Italy; \\ ${ }^{3}$ Institute of Pathological Anatomy, Polytechnic University of the Marche Region, Azienda Ospedaliero-Universitaria Ospedali \\ Riuniti, Ancona, Italy; \\ ${ }^{4}$ Institute of Dermatology, Polytechnic University of the Marche Region, Azienda Ospedaliero-Universitaria Ospedali Riuniti, \\ Ancona, Italy.
}

\begin{abstract}
Summary Objective: to investigate the role of $C D$ 44 immunohistochemical expression within tumoural and non-tumoural tissue, aiming to understand if it can help us to predict the need of performing inguinal lymph nodes dissection to complete surgery of the penis.

Materials and methods: CD44 immunohistochemical expression was investigated in tissue specimens from 39 patients with squamous cell carcinoma of the penis who underwent partial or total penectomy between 1987 and 2008. Patient age, tumour size, and grade; CD44 intensity score, cytological expression, topographic and distribution pattern were evaluated by immunohistochemistry on archived material and correlated with disease-specific survival.

Results: mean patients age was 67.7 years; mean followup was 130.44 months. Bilateral inguinal lymphadenectomy was performed in 14 patients; there were $8 \mathrm{N+}$ patients (23.5\%). pTis-pT1 vs. > pT1 and the EAU classification of risk group resulted to be predictive of lymph nodal metastases at univariate analysis (respectively $p=$ 0.006 and $p=0.045)$, but not the grading. The intensity score, cytological expression, topographic and distribution pattern of CD44 staining did not correlate with stage, grade and lymph nodes metastases. All disease related deaths occurred only in patients showing an high CD44 intratumoral expression, but this correlation is not statistically significant. Multivariate analysis showed that only lymph node metastasis was an independent prognostic factor predictive of lymph nodes metastases.

Conclusions: CD44 expression in patients with squamous cell carcinoma of the penis is not able to predict the need of performing inguinal lymphadenectomy; staging and the EAU classification of risk group resulted to be predictive of lymph nodal metastases.
\end{abstract}

KEY WORDS: Penis; Squamous cell carcinoma; CD44.

Submitted 3 March 2015; Accepted 15 March 2015

\section{INTRODUCTION}

Although squamous cell carcinoma of the penis is a rare disease, it is affected by an high mortality; many literature reports have pointed out that the presence of lymph nodal metastases are a determinant factor for disease specific survival (1); however, the correct treatment and the timing for inguinal lymph nodes dissection is still under debate. A number of studies are looking for prognostic factors able to distinguish patients who may benefit from inguinal lymph node dissection.

In many fields of clinical oncology attention has been given to angiogenesis and lymphangiogenesis, that are biological processes induced by tumoural cells able to give neoplastic spread in blood and lymphatic vessels.

Cluster of differentiation 44 (CD44), a principal cell surface receptor for hyaluronic acid, has been implicated in tumourigenesis and metastasis. CD44 is a glycoprotein encoded on the short arm of the chromosome 11 (2). The genetic sequence can encode for a variety of different proteins by selecting certain exons within the sequence, that is, the gene contains constants and variable exons; it exists as a standard $90 \mathrm{kDa}$ form as well as several CD44 variant isoform produced through alternative splicing; it is a cell membrane molecule that was first identified on lymphocytes and was initially found to have cell adhesion and cell homing functions. Since its initial discovery, the antigen has been identified in most human tissues and has been found to have a multiplicity of functions (3).

CD44 has been defined as cancer-initiating cell marker in many tumour entities (4); the results from initial preclinical trials enforce the multiple benefits that cancerinitiating cells receive from CD44 and strengthen the point that their function might be disrupted by the blockade of this molecule.

CD44 has also been implicated in metastasis.

In this study, we evaluated a cohort of squamous cell penile carcinoma to investigate the role of CD-44 immunohistochemical expression within tumoural and non- 
tumoural tissue, aiming to understand if it can help us to predict the need of performing inguinal lymph nodes dissection to complete surgery of the penis.

\section{Materials AND MEtHOdS}

\section{Patients}

Thirty-nine patients (mean age 67.7 years, range $50-88$ ) with squamous cell penile carcinoma, operated on by partial or total penectomy at our Institute of Urology between 1987 and 2008, were retrospectively evaluated. Of all the patients, 14 underwent to bilateral standard radical inguinal lymphadenectomy, performed in case of high clinical stage and grade (> T2 and/or > G3); in 2 of them palpable lymph nodes were detected.

Archived materials containing histological sections from patients were retrieved from the Institute of Pathological Anatomy and used for the study purpose. Histological grade was based on Broders' classification (5). Tumor staging was based on the TNM system (6).

Positive lymph nodes were defined as the presence of histologically confirmed lymph nodes metastases in patients who underwent inguinal lymphadenectomy. The features considered in this study were patient age, tumour size and grade, CD44 intensity score, CD44 cytological expression, CD44 topographic pattern and CD44 distribution pattern. Staging and EAU classification of risk groups were also considered (7).

All the considered parameters were correlated with patient specific survival.

\section{IMMUNOHISTOCHEMISTRY}

Immunohistochemistry was performed on conventional $5 \mu \mathrm{m}$ thick histological paraffin-embedded tissue sections on poly-L-lysine-coated glass slides. After heat-drying, sections were deparaffinized in xylene and sequentially rehydratated in gradients of ethanol. To better unmask antigenic sites, sections were treated with TUF solution (Histoline Laboratories, Milano, Italy) at $90^{\circ} \mathrm{C}$ for $10 \mathrm{~min}$ and incubated overnight at $4^{\circ} \mathrm{C}$ with the antibody CD44 (prediluted, Signet Laboratories Inc., Dedham, MA, USA). The reaction was revealed using the secondary antibody and streptoavidin-biotin-peroxidase technique (Dako-LSAB peroxidase kit, Dako-cytomation, Carpinteria, CA). After incubation with 3.3 diaminobenzidine (0.05 diaminobenzidine in $0.05 \mathrm{M}$ Tris buffer, pH 7.6 and $0.01 \%$ hydrogen peroxide), sections were counterstained with Mayer's Hematoxylin, coverslipped with Paramount and observed using a light microscope. Positive controls were represented by paraffin-embedded sections, previously shown to react with primary antibodies, from gastric carcinomas. For negative controls primary antibodies were replaced with nonimmune sera; sections of human tonsil tissue and of squamous cell carcinoma of uterine cervix were used as positive controls for the reaction against anti-CD44.

CD44 staining was assessed independently by

Table 2. two different operators (G.L., R.M.) counting individual microvessels on 10 fields at 200x magnification, i.e. a 20x objective lens and 10x ocular lens with $0.22 \mathrm{~mm}^{2}$ per field, on the invasive components of squamous cell carcinoma and normal epithelium.

The intensity of intracellular expression has been semiquantitatively evaluated according to a 4 point scale: negative, low, medium and high; the cytological expression has been considered in the membrane and cytoplasm; the topographic distribution has been divided on the basis of positivity in different cellular layers: basal, epibasal, superficial and diffuse; the distribution pattern has been divided in focal and spread.

\section{Statistical analysis}

Statistical analysis was performed using the KolmogorovSmirnov normality test for all the considered parameters. The $\chi 2$ test, Mann-Whitney $U$ and Kruskal-Wallis tests were used to compare nonparametric data. The values of CD44 staining were analysed with a model of classification by the ROC curve to characterize sensibility, specificity and area under the curve to predict lymph node metastases. Kaplan-Meier curves were designed to compare survival parameters, the influence of each parameter on survival was assessed using Cox proportional hazard models. The statistical significance has been considered with $p<0.05$ with two tails. Statistical analysis were performed with SPSS 16 and Graph-pad Prism 5.0.

\section{Table 1}

Clinicopathologic features of tumors (34 patients).

\begin{tabular}{|lc|c|}
\hline Variable & & Pts. (\%) \\
\hline Stage & Ta & $3(8 \%)$ \\
& Tis & $1(2.9)$ \\
& T1 & $14(41.2)$ \\
& T2 & $12(35.3)$ \\
& T3 & $3(8.8)$ \\
T4 & $1(2.9)$ \\
\hline Grade & G1 & $21(61.8)$ \\
& G2 & $7(20.6)$ \\
& G3 & $6(17.6)$ \\
\hline Nodes & N0 & $10(29.4)$ \\
& N+8 & $(23.5)$ \\
& NX & $16(47.1)$ \\
\hline
\end{tabular}

CD44 characteristics in normal and tumoural tissue (chi-square).

\begin{tabular}{|lc|c|c|c|}
\hline & & Normal & Tumoral & p \\
\hline CD44 Intensity score & Absent & $2(7,7)$ & $3(6.9)$ & 0.218 \\
& Low & $5(19,2)$ & $3(6.9)$ & \\
& Medium & $8(30,8)$ & $7(20.7)$ & \\
& High & $11(42,3)$ & $21(65.5)$ & \\
\hline CD44 Cytological expression & Membrane & $18(75)$ & $27(85.2)$ & 0.334 \\
& Cytoplasm & $6(25)$ & $7(14.8)$ & \\
\hline CD44 Topographic pattern & Basal & $3(12,5)$ & $9(25.9)$ & 0.836 \\
& Basal-epibasal & $20(83,3)$ & $20(63)$ & \\
& Epibasal & $1(4,2)$ & $5(11.1)$ & \\
\hline CD44 Dstribution pattern & Focal & $6(25)$ & $12(33.3)$ & 0.211 \\
& Spread & $18(75)$ & $22(66.7)$ & \\
\hline
\end{tabular}


Table 3.

CD44 characteristics in cancer tissue of patient without and with nodal metastases (chi-square test).

\begin{tabular}{|lc|c|c|c|}
\hline & & N0 & N+ & p \\
\hline CD44 Intensity score & Medium & $4(80 \%)$ & $1(20 \%)$ & 0.198 \\
\hline CD44 Cytological expression & Hembrane & $4(44.4 \%)$ & $5(55.6 \%)$ & \\
& Cytoplasm & $0(01.5 \%)$ & $5(38.5 \%)$ & 0.231 \\
CD44 Topographic pattern & Basal & $4(80 \%)$ & $1(100 \%)$ & \\
& Basal-epibasal & $4(50 \%)$ & $4(50 \%)$ & 0.277 \\
& Epibasal & $0(0 \%)$ & $1(100 \%)$ & \\
\hline CD44 Dstribution pattern & Focal & $4(66.7 \%)$ & $2(33.3 \%)$ & 0.533 \\
& Spread & $4(50 \%)$ & $4(50 \%)$ & \\
\hline
\end{tabular}

versus 38\%; P b .001, Wilcoxon test). When evaluating patients survival, we could observe that all disease related deaths occurred only in patients showing an high CD44 intratumoral expression; but this correlation is not statistically significant (log rank p = 0.146) (Figure 2). At univariate analysis, the presence of lymph nodes metastases resulted to be a risk factor for lower specific disease survival ( $p=0.014$, longrank test) (231.6 months vs. 71.5). Multivariate analysis with the

Cox proportional model showed that only lymph node metastasis was an independent prognostic factor.

\section{RESULTS}

Of the 39 patients, 5 were lost to follow-up. The mean age of the remaining 34 patients was 67.7 years (range, 5088 years); mean follow-up was 100.44 months (range, 14-243 months). The clinicopathological features of the tumours are shown in Table 1. No patient had distant metastases at the time of the diagnosis of the primary tumour. Bilateral inguinal lymphadenectomy was performed in 14 patients; there were $8 \mathrm{~N}+$ patients (23.5\%). None of the patients with non-palpable inguinal lymph nodes at diagnosis developed metastases (NO); for statistical purposes, they were therefore considered as N0. pTis-pT1 vs. > pTl and the EAU classification of risk group resulted to be predictive of lymph nodal metastases at univariate analysis (respectively $\mathrm{p}=0.006$ and $\mathrm{p}=0.045$ ), but not the grading,

\section{CD44 staining}

CD44 immunostaining was evaluated in cells in tumoural and normal tissue (Figure 1A-C); a positivity has been detected also in normal tissue, while in 3 patients a positivity was not detected in the tumour (Table 2); the intratumoral expression of CD44 has been observed mainly on cytoplasmic membrane (85.2\%). We can observe that there is no statistically significant difference in normal and tumoural tissue when considering intensity score, cytological expression, topographic and distribution pattern.

In Table 3 the CD44 characteristics in cancer tissue of patient without and with nodal metastases are shown; there was no statistically significant difference in cancer tissue between patients with non-metastatic and metastatic lymph nodes when considering intensity score, cytological expression, topographic pattern and distribution pattern. The intensity score, cytological expression, topographic and distribution pattern of CD44 staining did not correlate with stage, grade and lymph nodes metastases.

\section{Survival analysis}

Mean 5-year disease-specific survival was 71\%, with significant differences between N0 and $\mathrm{N}+$ patients $(96 \%$
Figure 2.

Patients survival in relation to CD44 intensity score $(p=0.1471)$.

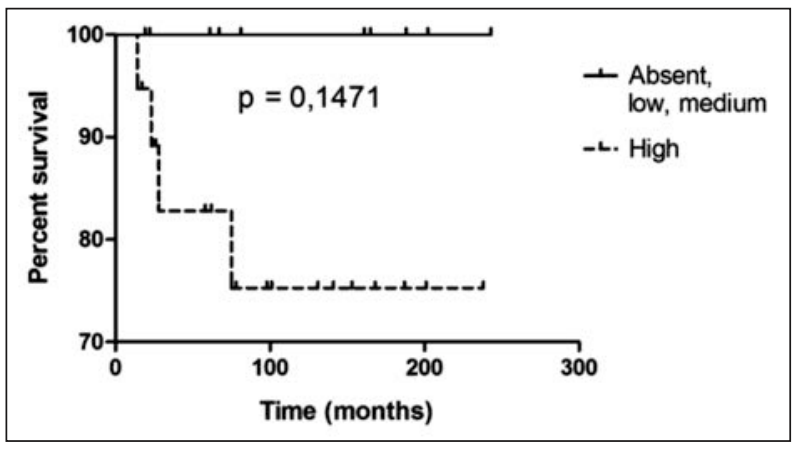

\section{Discussion}

CD44 is a highly heterogeneous transmembrane glycoprotein that is involved in the growth and metastasis of many types of cancer, acting as a cellular adhesion molecule.

In 1989, Stamenkovic found that a variety of carcinoma cell lines and solid tumours expressed the CD44 gene (8). In 1991, Günthert discovered that an isoform of CD44, when inserted into the genetic sequence of a non-metastasising tumour, gave it metastatic properties (9); these initial discoveries indicating that CD44 was involved in the metastatic process led to a large amount of research into the possible mechanisms and the degree of involvement of CD44. Guo showed that monoclonal 
antibodies specific for CD44 can completely inhibit the binding of cells of a human melanoma cell line and also inhibit the metastatic capability of the tumour cell in vivo (10); in addition, if the monoclonal antibody to CD44 was given seven days after tumour inoculation, the primary tumour continued to grow, although the tumour's metastatic capability was completely inhibited. Hoffman noted that the expression of CD44 by rat embryonic fibroblast cells correlated with metastatic tumour behaviour (11).

CD44 realizes its functions through multiple mechanisms, including inhibiting expression of Fas (12), activating the mitogen-activated protein kinase (MAPK) signalling pathway (13), binding to hyaluronic acid and regulating the activity of matrix metalloproteinases (MMPs) (14)

The presence of lymph nodes metastases in penile squamous cell carcinoma probably represents the most important prognostic factor for these patients.

EAU guidelines, regarding indications to inguinal lymphadenectomy in penile carcinoma, provide a stratification in 3 risk groups: low risk (Tis, pTa G1-G2, pT1 G1), intermediate risk (pTl G2) and high risk (any G3 and $>$ pT1) (7). Nodal dissection is therefore recommended in patients with palpable inguinal lymph nodes or in those with unfavourable clinic and pathologic characteristics; this is based upon the need to select those patients who will undergo surgery, eventually missing those patients with non-palpable lymph nodes but who may present micro-metastases.

It has already been observed that in $50-60 \%$ of patients palpable inguinal lymph nodes are only reactive (57\% in our series), while in up to $20 \%$ non-palpable lymph nodes harbor occult metastases (15). Furthermore, it is known that even the sentinel lymph node technique, performed by lymphography, is affected by $16 \%$ false negative results (16)

Therefore it has been looked for new prognostic factors for lymph nodes metastases.

Several authors have already looked for a pathologic or molecular marker able to predict lymph nodal metastases. In a series of 125 patients, Campos et al. (17) studied prognostic factors linked to lymphatic diffusion, particularly E-Cadherin and metalloproteinases 2 and 9.

E-Cadherin, a cell adhesion molecule, is involved in the mechanisms of diffusion in many tumors, low levels being correlated with metastases $(p=0.032)$; increased levels of metalloproteinases, enzymes able to cleave the basal membrane collagen, are correlated to disease recurrence. Lopez et al. have studied $\mathrm{p} 53$ protein in a series of 82 patients operated on of partial penectomy and bilateral lymphadenectomy, with a mean follow up of 88.7 months, and showed that the nuclear storage of p53 was correlated with nodal involvement and that the absence of $\mathrm{p} 53$ brings out a better 5 and 10 years specific survival (18). Recently a group from the Shangai University confirmed the importance of p53, defining a significant role in determining the probability of nodal involvement in Tl patients (19).

The aim of our study was to identify a pathologic factor able to predict the presence of nodal metastases; to our knowledge, this is the first report about the use of CD44 in penile squamous cell carcinoma. In our study, we have observed high levels of CD44 tumoural expression (83.1\%); however, we were not able to detected any correlation between intensity of expression and tumour stage and grade. Patients with nodal metastases did not show an increased CD44 expression. Therefore, we can say that the study of CD44 expression in patients with squamous cell carcinoma of the penis is not able to predict the need of performing inguinal lymphadenectomy. In our study, staging (pTis-pT1 vs. > pT1) and the EAU classification of risk group resulted to be predictive of lymph nodal metastases at univariate analysis (respectively $\mathrm{p}=0.006$ and $\mathrm{p}=0.045$ ), but not the grading; however the small number of cases does not allow us to draw definitive conclusions. By the univariate analysis of survival, the presence of nodal metastases represents the main influencing factor.

We have already studied the expression of D2-40 in patients with squamous cell carcinoma of the penis, and we have observed that it is a useful marker to predict node metastasis; in particular, low intratumoral LVD and overexpression of D2-40 in cancer cells were associated with lymph node metastasis and a worse clinical prognosis (20).

\section{Conclusions}

Based on our study, we can say that the CD44 expression in patients with squamous cell carcinoma of the penis is not able to predict the need of performing inguinal lymphadenectomy; staging and the EAU classification of risk group resulted to be predictive of lymph nodal metastases. The lack of a defined role of CD44 in our study may be mainly due to the small number of high stage cases, although relevant when considering that penile squamous cell carcinoma is a rare disease; our results need a confirmation by further multicentric studies including a greater number of cases.

\section{REFERENCES}

1. Ornellas AA, Nobrega BL, Wei Kin CE, et al. Prognostic factors in invasive squamous cell carcinoma of the penis: analysis of 196 patients treated at the Brazilian National Cancer Institute. J Urol. 2008; 180:1354.

2. Goodfellow PN, Banting G, Wiles MV, et al. The gene, MIC4, which controls expression of the antigen defined by monoclonal antibody F10.44.2, is on human chromosome 11. Eur J Immunol. 1982; 12:659.

3. Sneath RJS, Mangham DC. The normal structure and function of CD44 and its role in Neoplasia. J Clin Pathol: Mol Pathol 1998; 51:191.

4. Zöller M. CD44: can a cancer-initiating cell profit from an abundantly expressed molecule? Nat Rev Cancer. 2011; 11:254.

5. Broders AC. Squamous-cell epithelioma of the skin: a study of 256 casi. Ann Surg. 1921; 73:141.

6. Penis. In: Edge SB, Byrd DR, Compton CC, et al., eds.: AJCC Cancer Staging Manual. 7th ed. New York, NY: Springer, 2010, pp 447-55.

7. Solsona E, Algaba F, Horenblas S, et al. EAU Guidelines on Penile Cancer. Eur Urol. 46; 1:2004. 
8. Stamenkovic I, Amiot M, Pesando J, Seed B. A lymphocyte molecule implicated in lymph node homing is a member of the cartilage link protein family. Cell. 1989; 56:1057.

9. Günthert U, Hofmann M, Rudy W, et al. A new variant of glycoprotein CD44 confers metastatic potentials to rat carcinoma cells. Cell. 1991; 65:13.

10. Guo Y, Ma J, Wang J, et al. Inhibition of human melanoma growth and metastasis in vivo by anti-CD44 monoclonal antibody. Cancer Res. 1994; 54:1561.

11. Hofmann M, Rudy W, Günthert U, et al. A link between ras and metastatic behavior of tumor cells: ras induces CD44 promoter activity and leads to low-level expression of metastasis-specific variants of CD44 in CREF cells. Cancer Res. 1993; 53:1516.

12. Manabu Y, Yoshiya T, Koichi F, Yasumoto K. CD44 stimulation down-regulates Fas expression and Fas-mediated apoptosis of lung cancer cells. Int. Immunol. 2001; 13:1309.

13. Marhaba R, Bourouba M, Zoller M. CD44v6 promotes proliferation by persisting activation of MAP kinases. Cell Signal. 2005; 17:961.

14. En Kajita M, Itoh Y, Chiba T, et al. Membrane-type 1 matrix metalloproteinase cleaves CD44 and promotes cell migration. Cell Biol. 2001; 153:893.
15. Biedrzycki OJ, Hadway P, Cooke A, et al. Immunohistochemical analysis of negative inguinal lymph nodes in men with squamous cell carcinoma of the penis: are we missing micrometastases which could predict recurrence? BJU Int. 2006; 98:70.

16. Kroon BK, Horenblas S, Meinhardt W, et al. Dynamic sentinel node biopsy in penile carcinoma: evaluation of 10 years experience. Eur Urol. 2005; 47:601.

17. Campos RS, Lopes A, Guimaraes GC, et al. E-cadherin, MMP2, and MMP-9 as prognostic markers in penile cancer: analysis of 125 patients. Urology. 2006; 67:797.

18. Lopes A, Bezerra AL, Pinto CA, et al. p53 as a new prognostic factor for lymph node metastasis in penile carcinoma: analysis of 82 patients treated with amputation and bilateral lymphadenectomy. $J$ Urol. 2002; 168:81.

19. Zhu Y, Zhou XY, Yao XD, et al. The prognostic significance of p53, Ki-67, epithelial cadherin and matrix metalloproteinase-9 in penile squamous cell carcinoma treated with surgery. BJU Int. 2007; 100:204

20. Minardi $D$, d'Anzeo $G$, Lucarini $G$, et al. D2-40 immunoreactivity in penile squamous cell carcinoma: a marker of aggressiveness. Human Pathology. 2011; 42:1596.

\section{Correspondence}

Daniele Minardi, MD (Corresponding Author)

d.minardi@univpm.it

Giovanni Muzzonigro, MD

g.muzzonigro@univpm.it

Clinica Urologica, Università Politecnica delle Marche, A.O. Ospedali Riuniti Via Conca 71 - 60126 Ancona, Italy

Guendalina Lucarini, MD

guendalina.lucarini@univpm.it

Roberto Di Primio, MD

r.diprimio@univpm.it

Dipartimento di Scienze Cliniche e Molecolari- Istologia

Università Politecnica delle Marche, A.O. Ospedali Riuniti, Ancona, Italy

Oriana Simonetti, $M D$

o.simonetti@univpm.it

Istituto di Dermatologia, Università Politecnica delle Marche,

A.O. Ospedali Riuniti, Ancona, Italy

Rodolfo Montironi, MD

r.montironi@univpm.it

Istituto di Anatomia Patologica

Università Politecnica delle Marche, A.O. Ospedali Riuniti, Ancona, Italy 